\title{
Phase Composition at the Atomic-Size Scale through Multivariate Statistical Analysis of Atom Probe Tomography Data
}

\author{
M.R. Keenan ${ }^{1}$, V.S. Smentkowski ${ }^{2}$, R.M. Ulfig ${ }^{3}$, E. Oltman ${ }^{3}$, D.J. Larson ${ }^{3}$ and T.F. Kelly ${ }^{3}$ \\ ${ }^{1} 8346$ Roney Road, Wolcott, NY 14590 \\ ${ }^{2}$ General Electric Global Research Center, Niskayuna, NY 12309 \\ ${ }^{3}$ CAMECA Instruments, Inc., 5500 Nobel Drive, Madison, WI 53711
}

Atom probe tomography (APT), which uses time-of-flight mass spectrometry to reveal the specific identities of individual atoms at precise locations, would seem to produce data that is fundamentally univariate in nature. Initial approaches to applying multivariate statistical analysis techniques (MVSA) to APT data [1-3] overcame this limitation by partitioning the sample into a large number of volume elements (voxels) and combining all of the ions detected in each individual voxel into a corresponding mass spectrum. Typically, these voxels were on the order of $1 \mathrm{~nm}^{3}$ and contained several to a few tens of detected ions. Established MVSA techniques, which have proven effective for $3 \mathrm{D}$ time-of-flight secondary ion mass spectrometry images, were then applied to the aggregated data. While successful, this approach incurred the cost of degrading the high spatial resolution characteristic of atom probes. For a nominal resolution of $0.2 \mathrm{~nm}$, binning to just $1 \mathrm{~nm}^{3}$ implies a factor of 125 loss of resolution on a volumetric basis. In this presentation, we will demonstrate for the first time that MVSA techniques can be applied to APT data to estimate the chemical composition of a sample at the full spatial resolution of APT in three dimensions [4].

We will use simple arguments and simulations to suggest that MVSA can, in fact, be successfully applied to APT data aggregated to the native spatial resolution of the instrument. The arguments turn on the insight that while the atom probe only detects single ions, those can be considered random samples drawn from a local neighborhood defined by the spatial resolution of the instrument. Rather than predicting which specific atom is situated at a particular location, the multivariate model predicts the probabilities that atoms of any type are located there. In essence, the chemical-phase composition at the atomic-size scale is resolved.

Analyzing data binned to the size of a single atom $(\sim 0.2 \mathrm{~nm})$, poses severe challenges, both computational and conceptual, that test the limits of MVSA. The number of voxels (spectra) can easily reach hundreds of millions and the data sets are extremely sparse. It is important to note that despite the high detection efficiency of atom probes (up to 60\%), the typical spectrum at this size scale will contain either zero counts or only a single count. As the presence of inter-species correlations is essential to the operation of MVSA, we show that it is only necessary to consider the subset of spectra containing two or more counts each. While this is a small fraction of the total spectra, it is a large number in absolute terms allowing good statistical estimates to be made. In addition, efficient numerical approaches to performing principal component analysis (PCA) on these data sets are put forward, and it is shown that PCA can be computed in a couple of seconds on modestly sized computers for a dataset with over 50 million atoms, Figs. 1a and 1b. This sample consists of a two-phase nickel-base superalloy that has three significant principal components as determined by Poisson-scaled PCA. The three principal-component score images are shown in Fig. 1(c) as a color overlay. Besides the two expected phases, PCA reveals the presence of an interphase boundary as indicated by yellow in Fig. 1c. Fig. 2 displays the corresponding principal-component loadings, and Fig. 2c clearly shows that PC3 is associated with segregation of oxygen to the interphase boundary. Given the small number of oxygenated ions in the sample, the locations of, and correlations among these ions would be discovered only with difficulty in a typical manual analysis. 
References

[1] C.M. Parish, et al., Microsc. Microanal., 16 (Suppl 2) (2010) 1858-1859.

[2] M.R. Keenan et al., Microsc. Microanal., 16 (Suppl 2) (2010) 270-271.

[3] C.M. Parish \& M.K. Miller, Ultramicroscopy, 110 (11) (2010) 1362-1373.

[4] M.R. Keenan et al., Microsc. Microanal., submitted 2010.

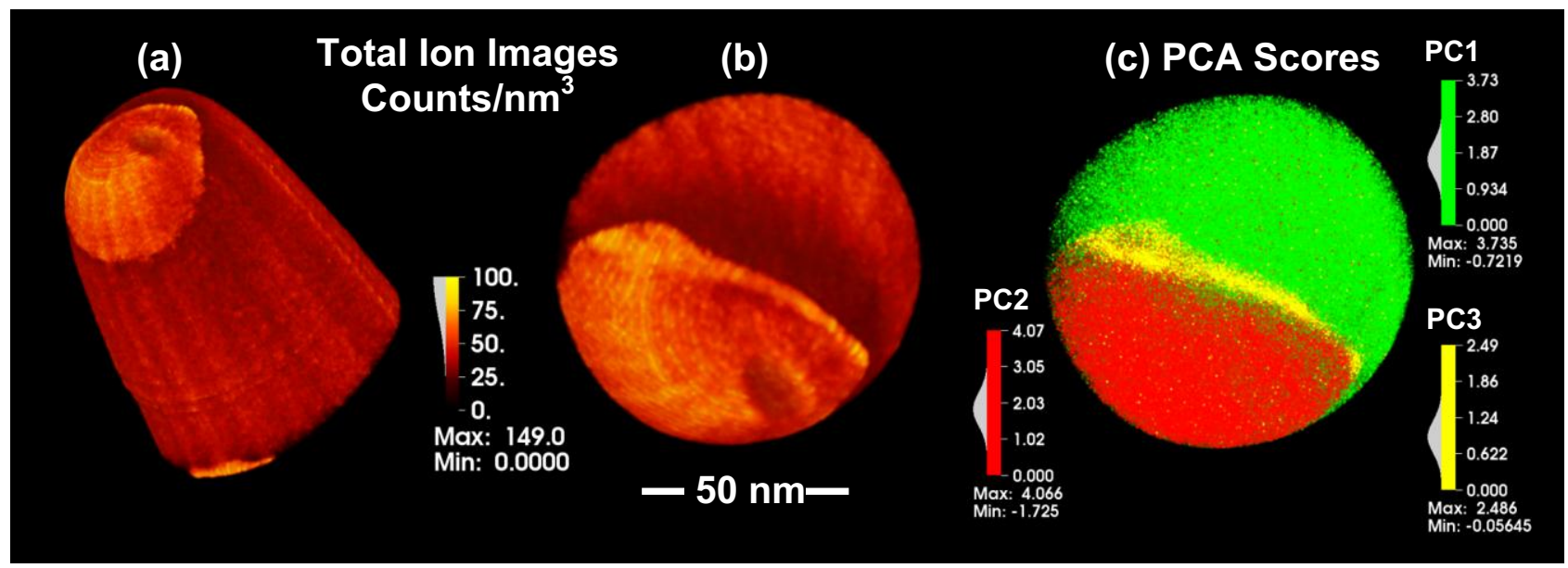

FIG. 1. APT analysis of a nickel-base superalloy two-phase region (a) Total ion image for the complete sample. (b) Image reoriented to look down the axis of the sample. (c) Three PCA scores obtained by the Poisson-scaled PCA method displayed as a color overlay in the same orientation.
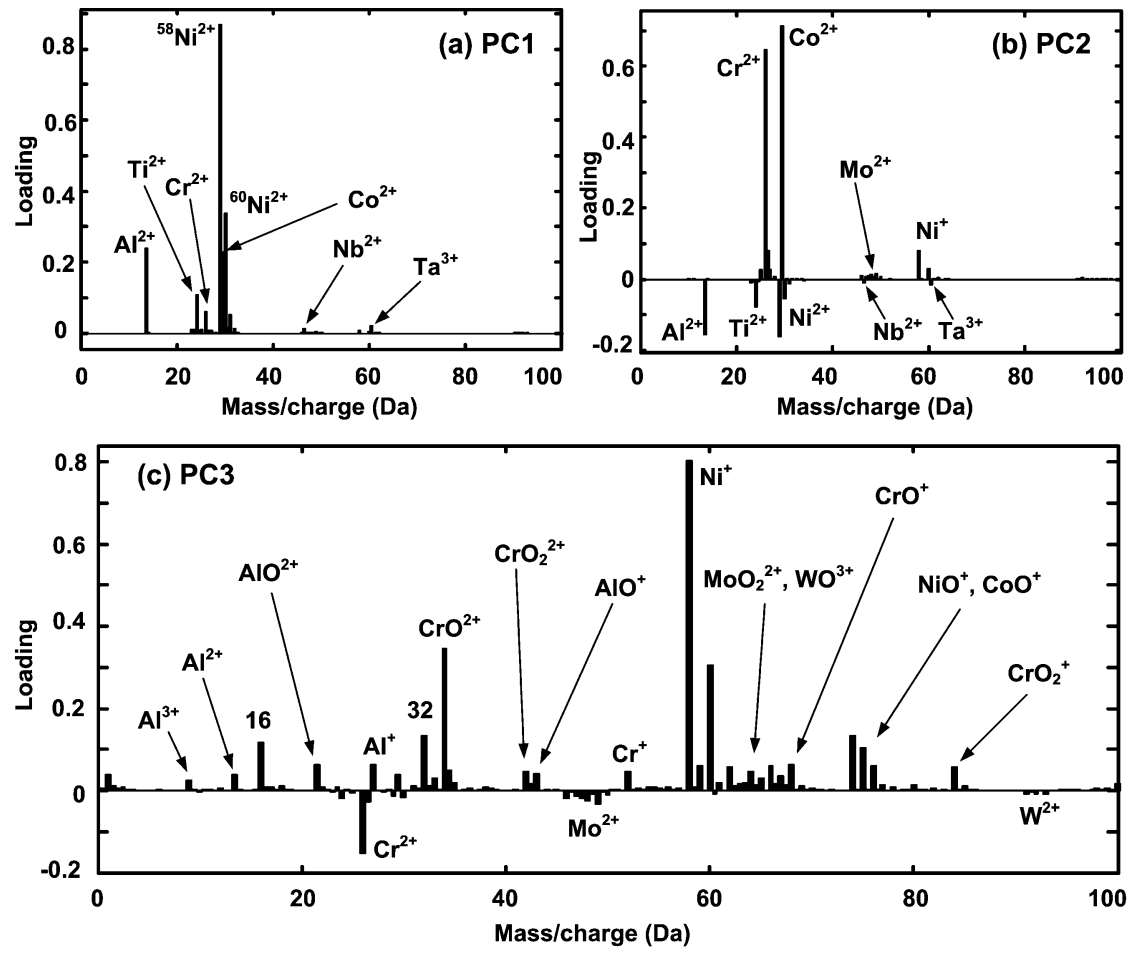

FIG. 2 The three principal-component loadings obtained by the Poisson-scaled PCA method for a nickel-base superalloy two-phase region. These correspond to the regions shown in Fig. 1. 